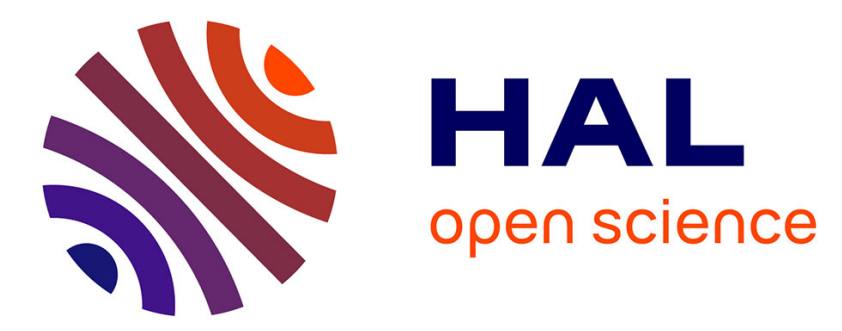

\title{
n-Order and maximum fuzzy similarity entropy for discrimination of signals of different complexity: Application to fetal heart rate signals
}

\author{
Amira Zaylaa, Souad Oudjemia, Jamal Charara, Jean-Marc Girault
}

\section{To cite this version:}

Amira Zaylaa, Souad Oudjemia, Jamal Charara, Jean-Marc Girault. n-Order and maximum fuzzy similarity entropy for discrimination of signals of different complexity: Application to fetal heart rate signals. Computers in Biology and Medicine, 2015, 64, 10.1016/j.compbiomed.2015.03.006 . hal01281250

\section{HAL Id: hal-01281250 \\ https://hal.science/hal-01281250}

Submitted on 1 Apr 2016

HAL is a multi-disciplinary open access archive for the deposit and dissemination of scientific research documents, whether they are published or not. The documents may come from teaching and research institutions in France or abroad, or from public or private research centers.
L'archive ouverte pluridisciplinaire HAL, est destinée au dépôt et à la diffusion de documents scientifiques de niveau recherche, publiés ou non, émanant des établissements d'enseignement et de recherche français ou étrangers, des laboratoires publics ou privés. 


\title{
n-order and maximum fuzzy similarity entropy for discrimination of signals of different complexity: application to fetal heart rate signals
}

\author{
Amira Zaylaa ${ }^{\mathrm{a}, \mathrm{c}}$, Souad Oudjemia ${ }^{\mathrm{b}}$, Jamal Charara ${ }^{\mathrm{c}}$, Jean-Marc Girault ${ }^{\mathrm{a}, *}$ \\ ${ }^{a}$ University François Rabelais of Tours, UMR Brain-Imaging, INSERM U930, Tours, France \\ ${ }^{b}$ University of Mouloud Mammeri, Tizzi Ouzou, Algeria \\ ${ }^{c}$ Department of Physics and Electronics, Faculty of Sciences, Lebanese University, Beirut, Lebanon
}

\begin{abstract}
This paper presents two new concepts for discrimination of signals of different complexity. The first focused initially on solving the problem of setting entropy descriptors by varying the pattern size instead of the tolerance. This led to the search for the optimal pattern size that maximized the similarity entropy. The second paradigm was based on the n-order similarity entropy that encompasses the 1-order similarity entropy. To improve the statistical stability, n-order fuzzy similarity entropy was proposed. Fractional Brownian motion was simulated to validate the different methods proposed, and fetal heart rate signals were used to discriminate normal from abnormal fetuses. In all cases, it was found that it was possible to discriminate time series of different complexity such as fractional Brownian motion and fetal heart rate signals. The best levels of performance in terms of sensitivity (90\%) and specificity (90\%) were obtained with the n-order fuzzy similarity entropy. However, it was shown that the optimal pattern size and the maximum similarity measurement were related to intrinsic features of the time series.
\end{abstract}

Keywords: Maximum similarity, n-order, Fetal heart rate, Entropy, Fuzzy, Complexity, fetal distress

10.1016/j.compbiomed.2015.03.006

\section{Introduction}

Detecting transitions in nonlinear dynamic systems is an important and challenging issue for which there is no definitive answer. This is particularly the case, for instance, in medicine when detecting the precise instant in time when a stroke occurs, i.e. when a patient goes from a normal to an abnormal state [1], or when discriminating between normal and abnormal fetuses on fetal heart rate recordings $[2,3]$.

One of the most promising ways for detecting such states lies in quantifying the complexity of time series. Among the possible complexity descriptors, fractal $[4,5,6,7]$, multifractal $[8,9,10,11,12]$, recurrent $[1,13]$ and entropy $[14,15,16,17,18]$ indicators are undoubtedly the most effective.

However, entropy descriptors are highly dependent on the setting parameters $(m, r)$ [19], and the choice of these parameters is critical, especially for moderately sized signal lengths [20]. Chon et al. [21] claimed that entropy descriptors such as the approximate entropy and sample entropy are not accurate in assessing signal complexity using the recommended values in the literature [22].

Though it has been suggested by [23] to set the tolerance $r$ empirically between 0.1 or 0.2 times the standard deviation of the time series, recent studies focusing on improving the detection of

\footnotetext{
${ }^{*}$ Corresponding Author: Jean-Marc Girault, Groupe Signal-Imagerie Eq5-U930 7 avenue Marcel Dassault 37200 Tours Cedex France; Email, jean-marc.girault@univ-tours.fr; Phone, +33 (0)2 47361081

Email addresses: amira.zaylaa@etu.univ-tours.fr (Amira Zaylaa), souad.oudjemia@etu.univ-tours.fr (Souad Oudjemia), jcharara@ul.edu.lb (Jamal Charara), jean-marc.girault@univ-tours.fr (Jean-Marc Girault)
} 
transitions $[24,19]$ have shown the interest of setting it at another value. This suggested value, adapted for the detection of transitions, has been proposed to maximise entropy $\mathcal{E}(m, r)$ :

$$
\left\{\begin{array}{l}
\mathcal{E}_{*}=\mathcal{E}\left(r^{*}, m\right) \\
r^{*}=\arg \max _{r}(\mathcal{E}(r, m),)
\end{array}\right.
$$

where $r$ is the tolerance and $m$ is the pattern size.

On the other hand, for the setting of pattern size $m$, few studies have focused on finding the optimal value of $m$, and the only studies published focused on the reconstruction of the original phase space $^{1}[25,26]$ rather than the detection of transitions or the discrimination of time series differing in complexity. However, the recent study by Restrepo et al. [19] tested several sizes of pattern $m$. Basically, the most commonly used values of $m$ were set at 1,2 or $3[27,3]$. These low values of $m$ were proposed because too high a value of $m$ leads to a poor estimation of the entropy $\mathcal{E}(m, r)[19]$ or to a poor reconstruction of the system dynamics.

Because entropy descriptors $\mathcal{E}(m, r)$ suffer from setting problems, and as there are no satisfactory solutions, the main purpose of this study was to extend the studies recently undertaken by $[24,21,19]$ based on the search for parameter settings leading to maximum entropy.

The first challenge to resolving the setting problems was investigating the value of $m^{*}$ to maximize entropy $\mathcal{E}(m, r)$ :

$$
\left\{\begin{array}{l}
\mathcal{E}^{*}=\mathcal{E}\left(r, m^{*}\right) \\
m^{*}=\arg \max _{m}(\mathcal{E}(r, m))
\end{array}\right.
$$

As this study was devoted only to searching for the optimal value $m^{*}$ with a constant value $r$, the latter was fixed at $r=0.2$ for the remainder of the study. The variable $r$ was therefore deliberately omitted from the subsequent equations. Moreover, the search for the optimal set of parameters $\left(r^{*}, m^{*}\right)$ to optimize the entropy $\mathcal{E}_{*}^{*}=\mathcal{E}\left(r^{*}, m^{*}\right)$ will be the subject of a future study. The study reported here showed that examining the role of the pattern size $m$ could provide important insights into quantifying the complexity of time series, thus leading to improved understanding of nonlinear dynamic systems. Furthermore, it was shown that the combined use of $m^{*}$ and $\mathcal{E}^{*}$ can provide a more consistent method to distinguish between different dynamics.

In view of the lack of a satisfactory method, the need to find a new transition detector or a discriminator is greatly needed. The second aim of this study was to establish a new paradigm to provide important insights into quantifying the complexity of time series. This new concept, for which, as suggested by [18], a membership function was introduced, encompassed the standard definition of entropy descriptors. Indeed, the general framework of "n-order fuzzy entropy" on which the new paradigm is based depends on the following equation:

$$
\mathcal{E}(n, m)=\phi(m+n)-\phi(m)
$$

where $n$ is the order, and $\phi$ is the average of the natural logarithm of the probability of finding similar patterns of size $m$. By setting $n=1$ in equation 1 , we recognized the definition of approximate entropy [14].

The concepts of maximum entropy and of n-order entropy were then introduced in the following work to achieve the above aims. The two new paradigms were validated through simulations and recordings of fetal heart rate.

\section{Concept of maximum Entropy}

\subsection{Theoretical aspects}

The aim of this section is to show that the concept of maximum entropy $\mathcal{E}^{*}=\mathcal{E}\left(m^{*}\right)$ is really valuable for discrimination purposes. For this purpose and within a more general framework, the

\footnotetext{
${ }^{1}$ The principle of reconstruction consists of finding the minimum embedding dimension $m$ that corresponds to a sudden change in the nearest false neighbors.
} 
similarity entropy was used as the foundation since its superiority recently was shown with regard to approximate entropy [18]. Definition of the similarity entropy is written as follows:

$$
\mathcal{E}_{p}(1, m)=\phi_{p}(m+1)-\phi_{p}(m)
$$

where

$$
\phi_{p}(m)=\frac{1}{N-(m-1)} \sum_{i=1}^{N-(m-1)} \log \left(\frac{1}{N-(m-1)} \sum_{j=1}^{N-(m-1)} D_{p}(i, j, m)\right) .
$$

The degree of similarity $D_{p}(i, j, m)$ between the $\mathbf{X}(i, m)$ and $\mathbf{X}(j, m)$ vectors within a fixed tolerance $r$, can be calculated through an exponential fuzzy function as follows:

$$
D_{p}(i, m, r)=e^{-\left(\frac{d(i, j, m)}{r}\right)^{p}} .
$$

When $p=2$ the membership function is a Gaussian function, whereas when $p=\infty$ the membership function is a Heaviside function. The distance $d(i, j, m)$ between the two $m$-patterns $\mathbf{X}(i, m)$ and $\mathbf{X}(j, m)$ is defined as follows:

$$
d(i, j, m)=d(\mathbf{X}(i, m), \mathbf{X}(j, m))=\max _{k \in(0, m-1)}|x(i+k)-x(j+k)|,
$$

where $x=x-\bar{x}$ and $\bar{x}$ stands for the mean of $x$. Thus a centered vector sequence $\mathbf{X}(m, i)$ is formed from a time series vector composed of $\mathrm{N}$ points:

$$
\mathbf{X}(m, i)=\{x(i), x(i+1), \ldots, x(i+m-1)\}-\overline{\mathbf{X}}(i, m),
$$

with

$$
\overline{\mathbf{X}}(i, m)=\frac{1}{m} \sum_{l=0}^{m-1} x(i+l) .
$$

To guarantee that the function $\phi_{p}(m)$ varies between 0 and 1 , a normalized version is proposed as follows:

$$
\Phi_{p}(m)=1+\frac{\phi_{p}(m)}{\log (N)},
$$

$N$ being the length of the time series.

Finally, 1-order entropy, that corresponds to the first discrete derivative of the function $\Phi_{p}(m)$ was reduced to:

$$
\mathcal{E}_{p}(1, m)=\Phi_{p}(m+1)-\Phi_{p}(m),
$$

and the search for $m^{*}$ maximizing the entropy $\mathcal{E}_{p}(1, m)$ and the maximum entropy $\mathcal{E}_{p}^{*}$ led to the following equations:

$$
\left\{\begin{array}{l}
m^{*}=\arg \max _{m}\left(\mathcal{E}_{p}(1, m)\right), \\
\mathcal{E}_{p}^{*}=\mathcal{E}_{p}\left(1, m^{*}\right)
\end{array}\right.
$$

with $p=\infty$ for non-fuzzy estimations and $p=2$ for fuzzy estimations.

Once theoretical aspects were complete it was then important to demonstrate the value of determining the maximum value of entropy $\mathcal{E}_{p}^{*}$ through simulations. These simulations were focused on fractional Brownian motion (fBm) and Lorenz time series because (i) they are good archetypes of biomedical signals and (ii) because their degrees of irregularity can easily be varied through the Hurst exponent $\mathcal{H}$.

It should be recalled that an $\mathrm{fBm}$ is a time series $x(t)$ governed by the self-affinity equation: $x(r t)=r^{\mathcal{H}} x(t)$, where $r$ is a scaling parameter and $\mathcal{H}$ the self-affine invariant called the "Hurst exponent". The autocorrelation function of self-affine time series is a decreasing function whose decreasing speed depends on the Hurst exponent: the higher the Hurst exponent, the more regular the time series and the longer the correlation length. 

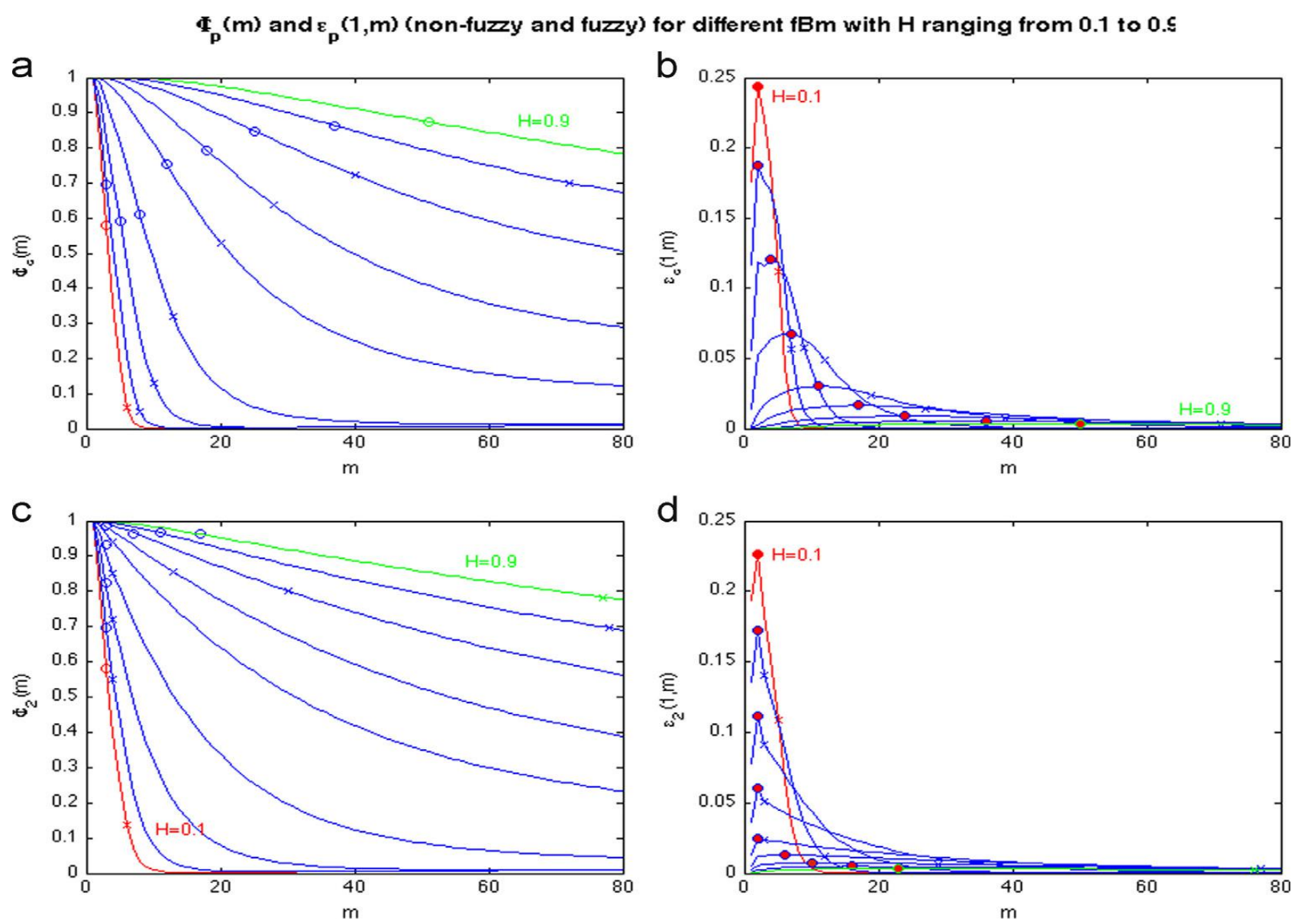

Figure 1: Complexity measurement for different fBm with $\mathcal{H}$ ranging from 0.1 to $0.9(r=0.2)$. (a) Non-fuzzy estimations with $\Phi_{\infty}\left(m_{\infty}\right)$. Blue circles correspond to inflection points. (b) Non-fuzzy estimations with $\mathcal{E}_{\infty}(1, m)$. Red dots correspond to maxima and blue crosses to inflection points. (c) Fuzzy estimations with $\Phi_{2}(m)$. Blue circles correspond to inflection points. (d) Fuzzy estimations with $\mathcal{E}_{2}(1, m)$. Red dots correspond to maxima and blue crosses to inflection points.

\subsection{Simulation results}

In order to show that $\mathcal{E}_{p}^{*}$ and $m^{*}$ can be related to the intrinsic features of fractional Brownian motion (fBm), 100 normalized fBms of unitary energy composed of $N=1024$ samples were simulated with Hurst exponents ranging from $\mathcal{H}=0.1$ to 0.9 . To guarantee that the maximum entropy was reached, the size of the pattern $\mathrm{m}$ was varied from 1 to 80 and $r=0.2$. The results are set out in Figs. 1 and 2 and Table 1.

Figs. 1a and 1c, represent the mean of $\Phi_{\infty}(m)$ and $\Phi_{2}(m)$. From these graphs, it can be seen that the $\Phi_{p}(m)$ functions that quantified the probability of finding patterns of size $m$ were monotonic, decreasing from 1 to 0 as $m$ increased. These functions that represented a cumulative effect possessed slopes depending on the Hurst exponent: the higher the Hurst exponent, the lower the slope. It can also be seen that $\Phi_{\infty}(m)$ and $\Phi_{2}(m)$ decreased as the size of the pattern increased. Indeed, the greater the size of the patterns, the lower the probability of finding big patterns. Fig. $1 \mathrm{~b}$ and $\mathrm{d}$, represent the mean of $\mathcal{E}_{\infty}(1, m)$ and $\mathcal{E}_{2}(1, m)$. From these graphs, it can be seen that the $\mathcal{E}_{p}(1, m)$ functions (cumulative effect removed), were non-monotonic, reaching a maximum $\mathcal{E}_{p}\left(1, m^{*}\right)$ and a location $m^{*}$ that both depended on the value of the Hurst exponent. These curves started from 0 and rose to a maximum and then decreased as $\mathcal{H}$ increased. As fBms are self-affine time series, their autocorrelations are decreasing functions whose correlation length depends on the Hurst exponent value: the lower the Hurst exponent value, the shorter the correlation length and the lower the pattern size $m$. Fig. $1 \mathrm{~b}$ and $\mathrm{d}$ shows $m$ values that are particularly interesting, the most visible are those maximizing the 1-order entropy. These values that depended on the Hurst exponent represented inflection points in the $\Phi_{p}(m)$ functions that were high-lighted in the $\mathcal{E}_{p}(1, m)$ 

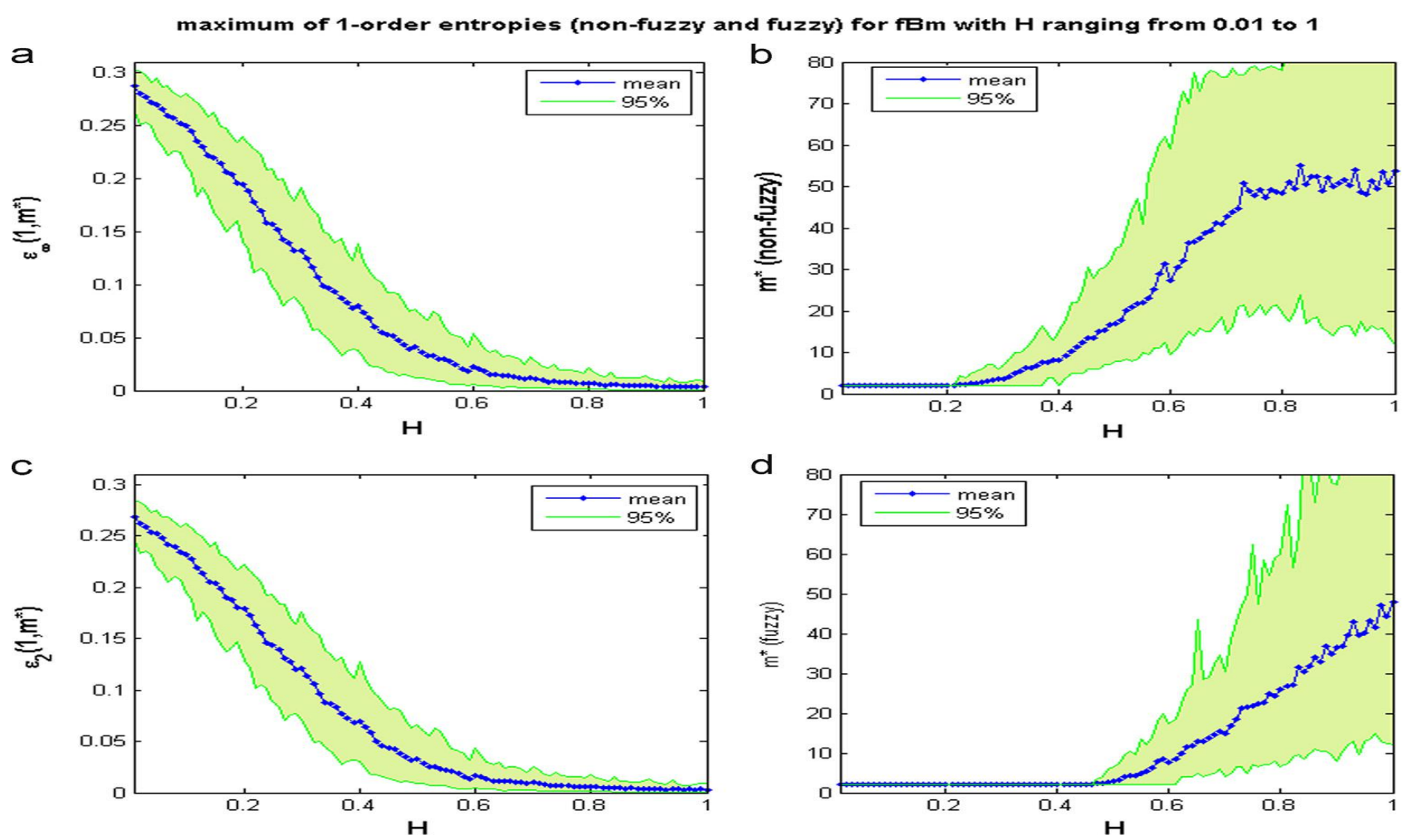

Figure 2: Maximum 1-order entropies (non-fuzzy and fuzzy) of $100 \mathrm{fBms}$ with $\mathcal{H}$ ranging from 0.0 to $1(\delta \mathcal{H}=0.01$, $r=0.2$ ). (a) $\mathcal{E}_{\infty}(\mathcal{H})$ (non-fuzzy estimation). Blue line corresponds to the mean estimation and green lines to $95 \%$ confidence interval. (b) $m^{*}(\mathcal{H})$ (non-fuzzy estimation). Blue line corresponds to the mean estimation and green lines to $95 \%$ confidence interval. (c) $\mathcal{E}_{2}^{*}(\mathcal{H})$ (fuzzy estimation). Blue line corresponds to the mean estimation and green lines to $95 \%$ confidence interval. (d) $m^{*}(\mathcal{H})$ (fuzzy estimation). Blue line corresponds to the mean estimation and green lines to $95 \%$ confidence interval.

functions representing the discrete derivatives of $\Phi_{p}(m)$. As inflection points can still be seen in the 1-order entropy $\mathcal{E}_{p}(1, m)$, then calculating high order derivatives of the functions $\Phi_{p}(m)$ should provide additional insight in the study of $\mathrm{fBm}$. This will be developed in the next section.

In order to advance in the examination of $\mathrm{fBm}$, new simulations were undertaken with Hurst exponents ranging from $\mathcal{H}=0.0$ to 1 by steps of $\delta \mathcal{H}=0.01$. $\mathcal{E}_{p}^{*}$ and $m^{*}$ were evaluated from 1-order entropies $\mathcal{E}_{p}(1, m)$ from each fBm with $p=\infty$ for non-fuzzy approaches and $p=2$ for fuzzy approaches. Fig. 2a and c represents the maximum of 1 -order entropies $\mathcal{E}_{\infty}^{*}(\mathcal{H})$ and $\mathcal{E}_{2}^{*}(\mathcal{H})$. The blue line corresponds to the mean estimation and green lines to $95 \%$ confidence interval obtained with the traditional normal-based approach. It can be seen from these graphs that $\mathcal{E}_{\infty}^{*}(\mathcal{H})$ and $\mathcal{E}_{2}^{*}(\mathcal{H})$ were monotonic functions, decreasing from $\approx 0.3$ to 0 as $\mathcal{H}$ increased, the value of $\approx 0.3$ being similar to that obtained for a Gaussian white noise, i.e. without any correlation in the time series. It can also be seen from these graphs that the higher the Hurst exponent $\mathcal{H}$, the lower the maximum 1-order entropy; indeed, the greater the regularity (degree of correlation), the lower the complexity. These graphs show clearly a link between the maximum 1-order entropy $\mathcal{E}_{p}^{*}(\mathcal{H})$ and the intrinsic parameter $\mathcal{H}$. Fig. $2 \mathrm{~b}$ and $\mathrm{d}$ represents the abscissa of the maximum 1-order entropies $m^{*}(\mathcal{H})$ (non-fuzzy and fuzzy). The blue line corresponds to the mean estimation and green lines to $95 \%$ confidence interval. It can be seen from these graphs that $m^{*}(\mathcal{H})$ were discrete monotonic functions, increasing from 2 to $\approx 50$ for non-fuzzy estimation and $\approx 40$ for fuzzy estimation as $\mathcal{H}$ increased: the higher the Hurst exponent $\mathcal{H}$, the greater the optimal size of pattern $m^{*}$. The latter corroborates the fact that the higher the Hurst exponent, the longer the correlation length and the greater the optimal pattern size. These graphs show clearly a link between the size of pattern $m^{*} \mathcal{H}$ and the intrinsic parameter $\mathcal{H}$.

As suggested in [3] Kruskal-Wallis tests with p-value $<5 \%$ were performed to validate that $\mathcal{E}_{p}^{*}$ and $m^{*}$ can be used for discrimination purposes. Kruskal-Wallis tests were performed between 


\begin{tabular}{cccccccccccc}
\hline $\mathcal{H}$ & 0.0 & 0.1 & 0.2 & 0.3 & 0.4 & 0.5 & 0.6 & 0.7 & 0.8 & 0.9 & 1.0 \\
$\delta \mathcal{H}_{\mathcal{E}^{*}}^{\dagger}$ (non-fuzzy) & 0.01 & 0.01 & 0.01 & 0.02 & 0.02 & 0.03 & 0.02 & 0.02 & 0.02 & 0.02 & 0.02 \\
$\delta \mathcal{H}_{\mathcal{E}^{*}}^{\dagger}$ (fuzzy) & 0.01 & 0.01 & 0.01 & 0.02 & 0.02 & 0.03 & 0.02 & 0.02 & 0.02 & 0.02 & 0.02 \\
$\delta \mathcal{H}_{m^{*}}^{\dagger}$ (non-fuzzy) & - & - & 0.02 & 0.02 & 0.02 & 0.03 & 0.02 & 0.02 & 0.02 & - & - \\
$\delta \mathcal{H}_{m^{*}}^{\dagger}$ (fuzzy) & - & - & - & - & - & 0.03 & 0.02 & 0.02 & 0.02 & 0.02 & 0.02 \\
\hline
\end{tabular}

Table 1: Kruskal-Wallis tests with p-value $<5 \%$ between $\mathcal{E}_{p}^{*(1)}$ and $\mathcal{E}_{p}^{*(2)}$, and $m^{*(1)}$ and $m^{*(2)}$. The Kruskal-Wallis test was performed from $\mathrm{fBm}$ with $\mathcal{H}$ ranging from 0 to 1 and $\delta \mathcal{H}$ ranging from 0.01 to 0.09 . First row: Hurst exponent value $\mathcal{H}$. Second row: minimum value $\delta \mathcal{H}_{\mathcal{E}^{*}}^{\dagger}$ ensuring a significant separation between two fBms of the Hurst exponent $\mathcal{H}$ and $\mathcal{H}+\delta \mathcal{H}$ with non-fuzzy approach. Third row: minimum value $\delta \mathcal{H}_{\mathcal{E}^{*}}^{\dagger}$ ensuring a significant separation between two fBms of the Hurst exponent $\mathcal{H}$ and $\mathcal{H}+\delta \mathcal{H}$ with fuzzy approach. Fourth row: minimum value $\delta \mathcal{H}_{m^{*}}^{\dagger}$ ensuring a significant separation between two fBms of the Hurst exponent $\mathcal{H}$ and $\mathcal{H}+\delta \mathcal{H}$ with non-fuzzy approach. Fifth row: minimum value $\delta \mathcal{H}_{m^{*}}^{\dagger}$ ensuring a significant separation between two fBms of the Hurst exponent $\mathcal{H}$ and $\mathcal{H}+\delta \mathcal{H}$ with fuzzy approach. Minimum values of $\delta \mathcal{H}^{\dagger}$ ensuring a significant separation between two fBms of Hurst exponents $\mathcal{H}$ and $\mathcal{H}+\delta \mathcal{H}$.

$\mathcal{E}_{p}^{*(1)}=\mathcal{E}_{p}^{*}(\mathcal{H})$ and $\mathcal{E}_{p}^{*(2)}=\mathcal{E}_{p}^{*}(\mathcal{H}+\delta \mathcal{H})$, and between $m^{*(1)}=m^{*}(\mathcal{H})$ and $m^{*(2)}=m^{*}(\mathcal{H}+\delta \mathcal{H})$. These tests were obtained from $\mathrm{fBm}$ with $\mathcal{H}$ ranging from 0.0 to 1 and with $\delta \mathcal{H}$ ranging from 0.01 to 0.09 . The minimum $\delta \mathcal{H}_{\mathcal{E}^{*}}^{\dagger}$ and $\delta \mathcal{H}_{m^{*}}^{\dagger}$ values above which the discrimination was significant are reported in Table 1. The results set out in Table 1 show that minimum $\delta \mathcal{H}_{\mathcal{E}^{*}}^{\dagger}$ and $\delta \mathcal{H}_{m^{*}}^{\dagger}$ deviations were dependent on the value of the Hurst exponent and varied from 0.01 to 0.03. It is obvious from Table 1 that $\mathcal{E}_{p}^{*}$ is a good discriminating parameter since it permits significant differentiation of two fBms differing by less than $10 \%$ of the Hurst exponent value. This outcome augurs well for discriminating two time series of different complexity. Findings regarding the other parameter $m^{*}$ were not so clear and suggest that it should be used with caution. Finally, it should be noted that the outcomes derived from $\mathcal{E}_{p}^{*}$ were very similar between non-fuzzy and fuzzy approaches, and this was not the case for $m^{*}$.

To sum up this section, it can be confirmed that the maximum amplitude $\mathcal{E}_{p}^{*}$ of the 1-order entropy was a good descriptor, directly related to intrinsic features of the time series under study, and was also a good indicator to discriminate different levels of complexity while keeping tolerance fixed at $r=0.2$. Although the maximum entropy augured well for the complexity analysis, not all features of the $\Phi_{p}(m)$ functions were used profitably. This latter point is the subject of the next section.

\section{Concept of n-order entropies}

\subsection{Theoretical aspects}

It was shown in the previous section that $\Phi_{p}(m)$ 's were monotonic decreasing functions whose slope depended on the Hurst exponent. This feature that was not used profitably is the starting point of this section. To benefit from the slope-dependence of $\Phi_{p}(\mathcal{H})$, the discrimination function was defined by:

$$
\Delta_{\mathcal{E} p}(n)=\left|\Phi_{p}^{(1)}(1+n)-\Phi_{p}^{(2)}(1+n)\right|,
$$

and was based on $\Phi_{p}^{(i)}(1+n)$ with $i=1$ for $\mathcal{H}$ and $i=2$ for $\mathcal{H}+\delta \mathcal{H}$. By slightly modifying the definition of $\Delta_{\mathcal{E} p}(n)$ as :

$$
\Delta_{\mathcal{E} p}(n)=\left|\left(\Phi_{p}^{(1)}(1+n)-\Phi_{p}^{(1)}(1)\right)-\left(\Phi_{p}^{(2)}(1+n)-\Phi_{p}^{(2)}(1)\right)\right|,
$$

and by imposing $\Phi_{p}^{(1)}(1)=\Phi_{p}^{(2)}(1)$ (made possible by the normalisation outlined in equation 7 ) the definition of the n-order similarity entropy previously presented in equation 1 was recognized with 
$m=1: \mathcal{E}_{p}^{(i)}(n, 1)=\Phi_{p}^{(i)}(1+n)-\Phi_{p}^{(i)}(1)$, leading to

$$
\Delta_{\mathcal{E} p}(n)=\Delta_{\mathcal{E} p}(n, 1)=\left|\mathcal{E}_{p}^{(1)}(n, 1)-\mathcal{E}_{p}^{(2)}(n, 1)\right| .
$$

Although it is probable that the following equation does not always guarantee $\Phi_{p}^{(1)}(m)=\Phi_{p}^{(2)}(m)$, equation 11 can be generalized $\forall m$ as follows:

$$
\Delta_{\mathcal{E} p}(n, m)=\left|\mathcal{E}_{p}^{(1)}(n, m)-\mathcal{E}_{p}^{(2)}(n, m)\right|,
$$

based on the n-order entropy $\mathcal{E}_{p}^{(i)}(n, m)$ defined by:

$$
\mathcal{E}_{p}^{(i)}(n, m)=\Phi_{p}^{(i)}(m+n)-\Phi_{p}^{(i)}(m)
$$

with $i=1$ for $\mathcal{H}$ and $i=2$ for $\mathcal{H}+\delta \mathcal{H} . p=\infty$ corresponded to non-fuzzy estimations and $p=2$ to fuzzy estimations.

All possible values of $\mathcal{E}_{p}^{(i)}(n, m)$ were gathered in the symmetrical matrix $\mathcal{M}_{p}^{(i)}$ defined by:

$$
\mathcal{M}_{p}^{(i)}=\left(\begin{array}{ccccc}
0 & \mathcal{E}_{p}^{(i)}(1,1) & \mathcal{E}_{p}^{(i)}(2,1) & \mathcal{E}_{p}^{(i)}(3,1) & \ldots \\
\mathcal{E}_{p}^{(i)}(1,1) & 0 & \mathcal{E}_{p}^{(i)}(1,2) & \mathcal{E}_{p}^{(i)}(2,2) & \ldots \\
\mathcal{E}_{p}^{(i)}(2,1) & \mathcal{E}_{p}^{(i)}(1,2) & 0 & \mathcal{E}_{p}^{(i)}(1,2) & \ldots \\
\mathcal{E}_{p}^{(i)}(3,1) & \mathcal{E}_{p}^{(i)}(2,2) & \mathcal{E}_{p}^{(i)}(1,3) & 0 & \ldots \\
\ldots & \ldots & \ldots & \ldots &
\end{array}\right)
$$

where $\mathcal{M}_{p}^{(i)}(k, l)=\left|\Phi_{p}^{(i)}(k)-\Phi_{p}^{(i)}(l)\right|$. This matrix was symmetrical since $\left|\Phi_{p}^{(i)}(k)-\Phi_{p}^{(i)}(l)\right|$ was equal to $\left|\Phi_{p}^{(i)}(l)-\Phi_{p}^{(i)}(k)\right|$. The structure of this matrix was specific since its main diagonal was null $\left(\mathcal{M}_{p}^{(i)}(k, k)=0\right)$. The main diagonal corresponded to the 0 -order entropies and the diagonals just above and below the main diagonal corresponded to the 1-order entropies (in blue), 2-order entropies(in green), 3-order entropies (in red), etc..

Finally, the discrimination function $\Delta_{\mathcal{M} p}$ based on the Matrix $\mathcal{M}_{p}^{(i)}$ was defined by:

$$
\Delta_{\mathcal{M} p}=\left|\mathcal{M}_{p}^{(1)}-\mathcal{M}_{p}^{(2)}\right|
$$

\subsection{Simulation results}

For discrimination purposes, 200 normalized fBms composed of 1024 samples were simulated: 100 fBms with $\mathcal{H}=0.07$ and 100 with $\mathcal{H}+\delta \mathcal{H}=0.3$ were selected because it had been shown in [12] that such fBms could coarsely model fetal heart rate signals for abnormal and normal signals, respectively. The size of the pattern $m$ varied from 1 to 30 to guarantee that the maximum was reached, and therefore $\Phi_{p}(m)$ and $\mathcal{E}_{p}(1, m)$, as well as a discrimination function $\Delta_{\mathcal{E} p}(n)$ were considered for these kinds of $\mathrm{fBm}$. The feasibility of this new concept was validated by the simulation results reported in Figs. 4, 5.

Figs. 3a-d represent $\Phi_{\infty}^{(1)}(m), \Phi_{\infty}^{(2)}(m), \mathcal{E}_{\infty}^{(1)}(n, 1), \mathcal{E}_{\infty}^{(2)}(n, 2), \mathcal{E}_{\infty}^{(1)}(1, m), \mathcal{E}_{\infty}^{(2)}(1, m), \Delta_{\mathcal{E}}(1, m)=$ $\mathcal{E}^{(1)}(1, m)-\mathcal{E}^{(2)(1, m)}$ and $\Delta_{\mathcal{E}}(n, 1)=\mathcal{E}^{(1)}(n, 1)-\mathcal{E}^{(2)(n, 1)}$. Figs. 3e-5h represent the latter functions with fuzzy estimations. It can be seen from $3 \mathrm{c}-\mathrm{d}$ and $\mathrm{g}$-h that location of the maximum $\Delta_{\mathcal{E} p}^{*}$ occurred at positions that were different from those obtained for $\mathcal{E}_{p}^{*(i)}$. Note that $\Delta_{\mathcal{E} p}^{*}\left(n^{*}, 1\right)$ was defined by Fig. 3d and h:

$$
\left\{\begin{array}{l}
\Delta_{\mathcal{E} p}^{*}=\max \left(\Delta_{\mathcal{E} p}(n, 1)\right) \\
n^{*}=\arg \max _{n} \Delta_{\mathcal{E} p}(n, 1)
\end{array}\right.
$$

Maximum $\Delta_{\mathcal{E} p}\left(1, m^{*}\right)$ was defined by Fig. $3 \mathrm{~d}$ and h:

$$
\left\{\begin{array}{l}
\Delta_{\mathcal{E} p}^{*}=\max \left(\Delta_{\mathcal{E} p}(1, m)\right), \\
m^{*}=\arg \max _{m} \Delta_{\mathcal{E} p}(1, m) .
\end{array}\right.
$$



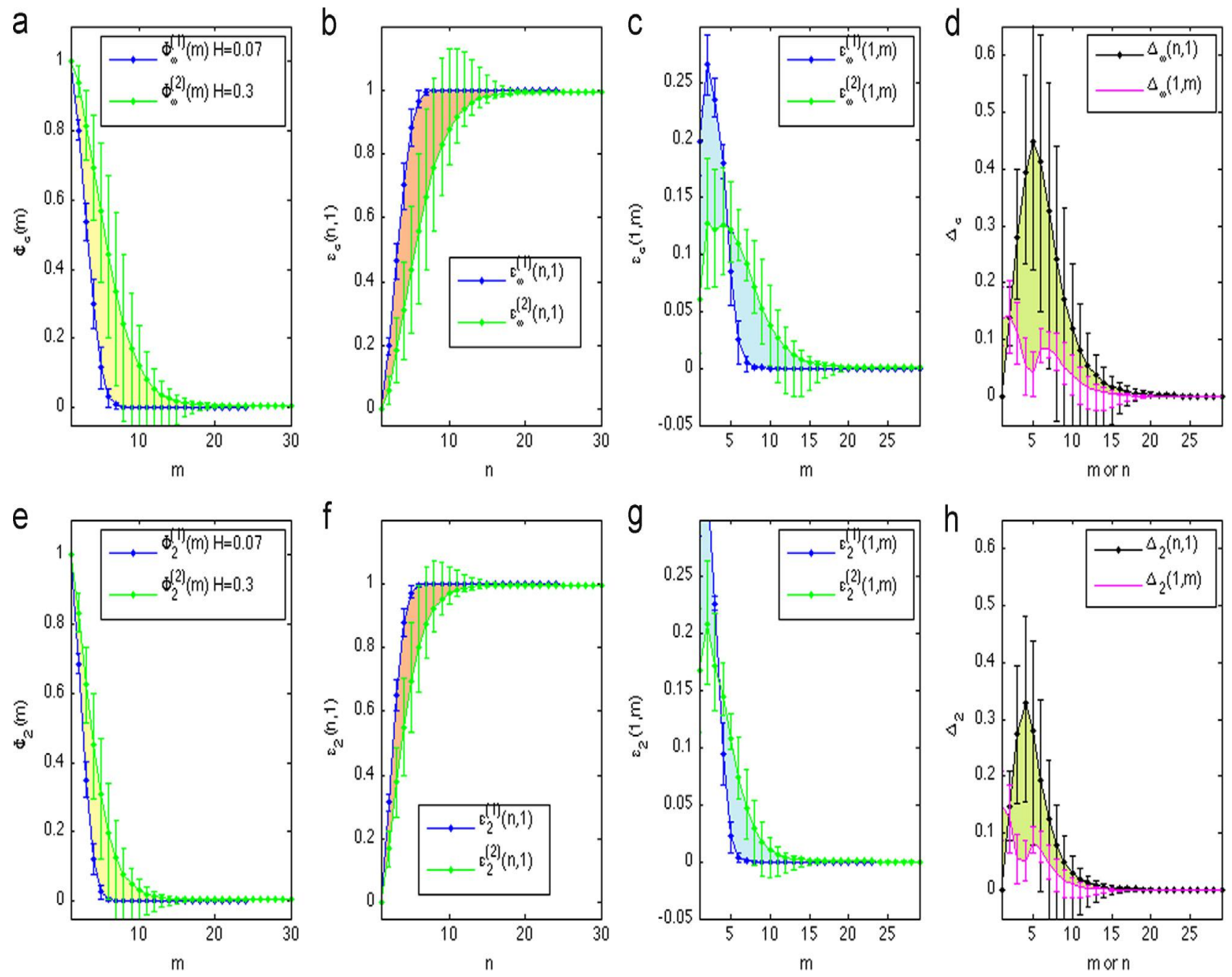

Figure 3: Complexity measurements for two different fBm with $\mathcal{H}=0.07$ and $\mathcal{H}+\delta \mathcal{H}=0.3,(r=0.2)$. Upper and lower bars correspond to $95 \%$ confidence interval, respectively. (a) Non-fuzzy estimations with $\Phi_{\infty}^{(1)}(m)$ (in blue) for $\mathcal{H}=0.07$ and $\Phi_{\infty}^{(2)}(m)$ (in green) for $\mathcal{H}+\delta \mathcal{H}=0.3$. Differences between $\Phi_{\infty}^{(1)}(m)$ and $\Phi_{\infty}^{(2)}(m)$ represented by the yellow area. (b) Non-fuzzy estimations with $\epsilon_{\infty}^{(1)}(n, 1)$ (in blue) and $\epsilon_{\infty}^{(1)}(n, 1) \mid$ (in green). Deviation between $\epsilon_{\infty}^{(1)}(n, 1)$ (in blue) and $\epsilon_{\infty}^{(1)}(1, m) \mid$ represented by orange area. (c) Non-fuzzy estimations with $\left|\mathcal{E}_{\infty}^{(1)}(1, m)\right|$ (in blue) and $\left|\mathcal{E}_{\infty}^{(2)}(1, m)\right|$ (in green). Differences between $\left|\mathcal{E}_{\infty}^{(1)}(1, m)\right|$ and $\left|\mathcal{E}_{\infty}^{(2)}(1, m)\right|$ represented by blue area. (d) Non-fuzzy estimation with $\Delta_{\mathcal{E} \infty}(n, 1)$ (in black) and $\Delta_{\mathcal{E} \infty}(1, m)$ (in magenta). Differences between $\Delta_{\mathcal{E} \infty}(n, 1)$ and $\Delta_{\mathcal{E}}(1, m)$ represented by green area. (e) Fuzzy estimations with $\Phi_{2}^{(1)}(m)$ (in blue) for $\mathcal{H}=0.07$ and $\Phi_{2}^{(2)}(m)$ (in red) for $\mathcal{H}=0.3$. (f) Fuzzy estimations with $\epsilon_{2}^{(1)}(n, 1)$ (in blue) and $\epsilon_{2}^{(2)}(n, 1)$ (in green). Differences between $\epsilon_{2}^{(1)}(n, 1)$ and $\epsilon_{2}^{(2)}(n, 1)$ are represented by orange area. (g) Fuzzy estimations with $\left|\mathcal{E}_{2}^{(1)}(1, m)\right|$ (in blue), $\left|\mathcal{E}_{2}^{(2)}(1, m)\right|$ (in green). (h) Fuzzy estimations with $\Delta_{\mathcal{E} 2}(n, 1)$ (in black) and $\Delta_{\mathcal{E} 2}(1, m)$ (in magenta). Differences between $\Delta_{\mathcal{E} 2}(n, 1)$ and $\Delta_{\mathcal{E} 2}(1, m)$ represented by green area.

It can be seen from these graphs that

$$
\Delta_{\mathcal{E} p}\left(n^{*}, 1\right)>\Delta_{\mathcal{E} p}\left(1, m^{*}\right)
$$

Indeed, $\Delta_{\mathcal{E} \infty}\left(5^{*}, 1\right)>\Delta_{\mathcal{E} \infty}\left(1,2^{*}\right)$ and $=\Delta_{\mathcal{E} 2}\left(4^{*}, 1\right)>\Delta_{\mathcal{E} 2}\left(1,2^{*}\right)$ were obtained and for $p=\infty$ and for $p=2$, respectively. This suggested that it was more advantageous to use $n$-order entropy rather than 1-order entropy.

Several values derived from Fig. 3 are reported in Tables 2 and 3.

The results derived from Table 2 showed the following:

- the lowest "relative accuracy" was obtained for $\mathcal{E}_{\infty}\left(5^{*}, 1\right)$ since it represented on average $(91 / 14) / 2=52.5 \%$ of the mean value while it represented $(95+22) / 2=58.5 \%$ and $(71+$ 
$109) / 2=90.0 \%$ for $\mathcal{E}_{\infty}\left(1,2^{*}\right)$ and $\Phi(5)$, respectively. This is advantageous for discrimination purposes.

- The lowest "relative accuracy" was obtained for fuzzy estimations compared to non-fuzzy estimations. Reductions off $(90-72)=18.0 \%,(52.5-32.5)=20 \%$ and $(58.5-31)=27.5 \%$ were obtained, respectively. This indicates that the fuzzy approach fully satisfies its role.

- By applying Kruskal-Wallis tests with a p-value $<5 \%$ to the complexity parameters obtained with $\mathcal{H}=0.07$ and with $\mathcal{H}+\delta \mathcal{H}=0.3$, the complexity parameters were significantly different.

\begin{tabular}{ccccccc}
\hline & Non-Fuzzy & \multicolumn{5}{c}{ Fuzzy } \\
\cline { 2 - 6 } $\mathrm{H}$ & $\Phi_{\infty}(5)^{a}$ & $\mathcal{E}_{\infty}\left(5^{*}, 1\right)^{a}$ & $\mathcal{E}_{\infty}\left(1,2^{*}\right)^{a}$ & $\Phi(4)^{a}$ & $\mathcal{E}_{2}\left(4^{*}, 1\right)^{a}$ & $\mathcal{E}_{2}\left(1,2^{*}\right)^{a}$ \\
\hline 0.3 & $0.37<0.57<0.77$ & $0.24<0.44<0.64$ & $0.07<0.13<0.18$ & $0.29<0.45<60$ & $0.40<0.55<0.70$ & $0.16<0.21<0.27$ \\
$(\mathrm{i}=2)$ & $(71 \%)$ & $(91 \%)$ & $(95 \%)$ & $(69 \%)$ & $(55 \%)$ & $(52 \%)$ \\
0.07 & $0.07<0.12<0.16$ & $0.82<0.88<0.93$ & $0.24<0.27<0.30$ & $0.07<0.12<0.16$ & $0.84<0.88<0.93$ & $0.32<0.34<0.36$ \\
$(\mathrm{i}=1)$ & $(109 \%)$ & $(14 \%)$ & $(22 \%)$ & $(75 \%)$ & $(10 \%)$ & $(12 \%)$ \\
\hline
\end{tabular}

Table 2: Average values in between the $95 \%$ confidence interval of specific values of $\Phi_{p}(m), \mathcal{E}_{p}(n, m)$ for two fBms with $i=1, \mathcal{H}=0.07$ and $i=2, \mathcal{H}+\delta \mathcal{H}=0.3$. Values in parentheses are a kind of "relative accuracy" that represents differences in values of confidence intervals normalized by the average value.

${ }^{a}$ The superscript $\dagger$ indicates that the complexity parameters obtained with $\mathcal{H}=0.07$ were significantly different from those obtained with $\mathcal{H}+\delta \mathcal{H}=0.3$ (Kruskal-Wallis tests with a p-value $<5 \%$

The results shown in Table 3 indicated the following:

- n-Order entropy was always higher than 1-order entropy since: $\Delta_{\mathcal{E} \infty}^{*}(5,1)>\Delta_{\mathcal{E} \infty}^{*}(1,2)$ for non-fuzzy estimations and $\Delta_{\mathcal{E} 2}^{*}(4,1)>\Delta_{\mathcal{E} 2}^{*}(1,1)$ for fuzzy estimations.

- The lowest "relative accuracy" was obtained for fuzzy estimations since it represented $98 \%$ of the mean value of $\Delta_{\mathcal{E} \infty}^{*}(5,1)$ and $86 \%$ of $\Delta_{\mathcal{E} \infty}^{*}(1,2)$ and $97 \%$ of $\Delta_{\mathcal{E} 2}^{*}(4,1)$ and $92 \%$ of $\Delta_{\mathcal{E} 2}^{*}(1,1)$. Once again, it was advantageous to use fuzzy estimations since the standard deviations were the smallest. However, it can be seen that the mean values obtained from fuzzy estimations were lower than those obtained from non-fuzzy estimations. This latter point was not in favor of fuzzy estimations when fBms were considered.

- By applying a Kruskal-Wallis tests with a p-value $<5 \%$ to complexity parameters obtained with $\mathcal{H}=0.07$ and with $\mathcal{H}+\delta \mathcal{H}=0.3$, the complexity parameters were significantly different.

\begin{tabular}{cccc}
\hline Non-Fuzzy & \multicolumn{3}{c}{ Fuzzy } \\
\hline$\Delta_{\mathcal{E} \infty}^{*}(5,1)^{a}$ & $\Delta_{\mathcal{E} \infty}^{*}(1,2)^{a}$ & $\Delta_{\mathcal{E} 2}^{*}(4,1)^{a}$ & $\Delta_{\mathcal{E} 2}^{*}(1,1)^{a}$ \\
$0.23<0.45<0.67$ & $0.08<0.14<0.20$ & $0.16<0.33<0.48$ & $0.09<0.15<0.21$ \\
$(98 \%)$ & $(86 \%)$ & $(97 \%)$ & $(92 \%)$ \\
\hline
\end{tabular}

Table 3: Average values and "relative accuracy" of discrimination functions $\Delta_{\mathcal{E}}(n, m)$ derived from FHR signals. Values in parentheses are a kind of "relative accuracy" that represents differences in values of confidence interval $(75 \%)$ normalized by the average value. Fuzzy estimations were valuable for reducing estimation fluctuations.

${ }^{a}$ The superscript $\dagger$ indicates that the complexity parameters obtained with $\mathcal{H}=0.07$ were significantly different from those obtained with $\mathcal{H}+\delta \mathcal{H}=0.3$ (Kruskal-Wallis tests with a p-value $<5 \%$

Moreover, if the idea was to discriminate two fBms (with for instance ${ }^{2} \mathcal{H}=0.3$ and $\mathcal{H}+\delta \mathcal{H}=$ $0.325)$ from any values of $m^{*}$ and $n^{*}$ except for $m^{*}$ and $n^{*}$, then it was more advantageous to use

\footnotetext{
${ }^{2}$ These values were used since the difference was significant whatever the values of $n$ and $m$ when considering $\mathcal{H}=0.07$ and $\mathcal{H}+\delta \mathcal{H}=0.3$
} 


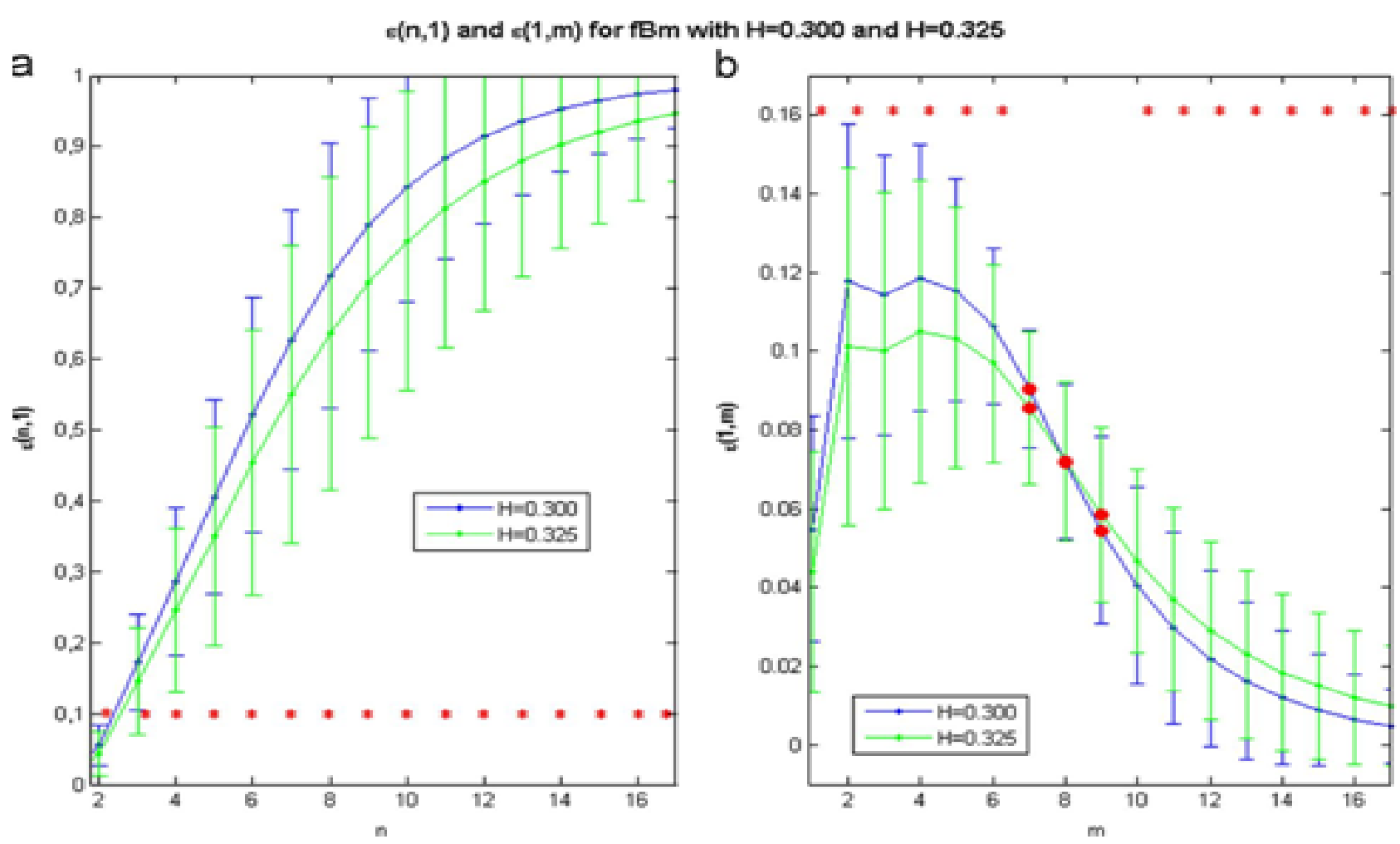

Figure 4: Complexity measurements $\mathcal{E}^{(1)}(n, 1), \mathcal{E}^{(2)}(n, 1), \mathcal{E}^{(1)}(1, m)$ and $\mathcal{E}^{(1)(n, 1)}$ for two different fBms with $\mathcal{H}=0.3$ and $\mathcal{H}+\delta \mathcal{H}=0.325(r=0.2)$, respectively. Upper and lower bars correspond to $95 \%$ confidence interval, respectively. (a) Kruskal-Wallis tests with p-value $<5 \%$ were performed between $\mathcal{E}^{(1)}$ and $\mathcal{E}^{(2)}$, * indicates that the difference was significant. (b) Kruskal-Wallis tests with p-value $<5 \%$ were performed between $\mathcal{E}^{(1)}$ and $\mathcal{E}^{(2)}$, * indicates that difference was significant. )

n-order entropy than 1-order entropy (Fig. 4) since the difference between two fBms was significant for n-order entropy whatever the value $n$, whereas the difference for 1-order entropy was significant whatever the value of $m$ except for $m=7,8,9$.

$\mathcal{M}_{p}^{(1)}$ and $\mathcal{M}_{p}^{(2)}$ are reported in Figs. $5 \mathrm{~b}$ and $5 \mathrm{c}$ for non-fuzzy estimations and for fuzzy estimations they are reported in Figs. $5 \mathrm{f}$ and $5 \mathrm{~g}$. It can be seen from Figs. $5 \mathrm{~b} 5 \mathrm{c}, 5 \mathrm{f}$ and $5 \mathrm{e}$ that the more interesting values were located on the border of the matrix, i.e. for small values of $n$ when $m$ was fixed and vice versa. The matrix difference $\Delta_{\mathcal{M} p}$ reported in Fig. $5 \mathrm{~d}$ for non-fuzzy estimations and in Fig. 5h for fuzzy estimations, showed that the best values were between $n=4$ and 8 when $m=1$ and between $n=25$ and 30 when $m=6$. These results indicate that it is worthwhile using the n-order entropy for discriminating fBms of different levels of complexity.

To sum up this section, it was shown that it was more advantageous to use maximum n-order entropy rather than maximum 1-order entropy. This was confirmed for high values of entropy and for the lowest relative accuracy. However, these results were obtained for fBms that were hypothesized as a coarse model of fetal heart rate signals. In order to confirm that n-order entropy still works on biomedical signals, examination of fetal heart rate signals is reported in the following section.

\section{Discrimination of fetal heart rate time series}

The aim of this section is to demonstrate the validation that the n-order fuzzy entropy provides discrimination between abnormal and normal fetuses. Data collection is introduced first and then the methods are compared.

\subsection{Data collection}

Fetal heart rate (FHR) signals were measured using a homemade pulse Doppler system codeveloped with Altaïs Technologies, (Tours, France). This system which transmits ultrasound waves 
a
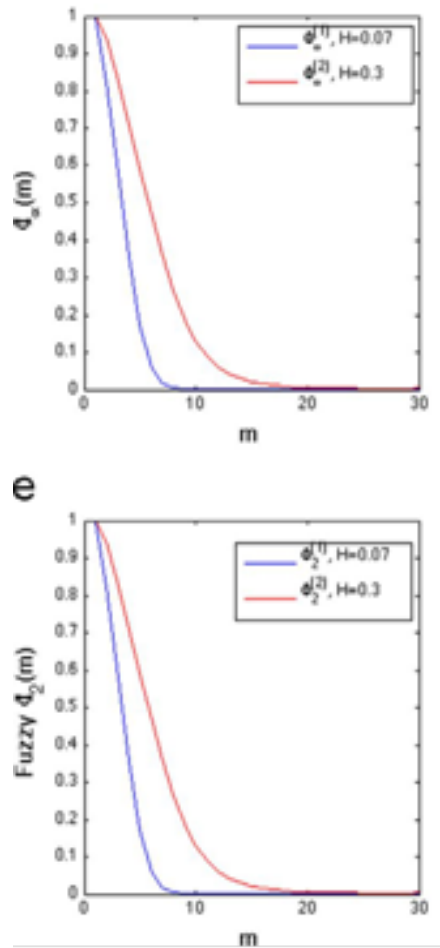

b

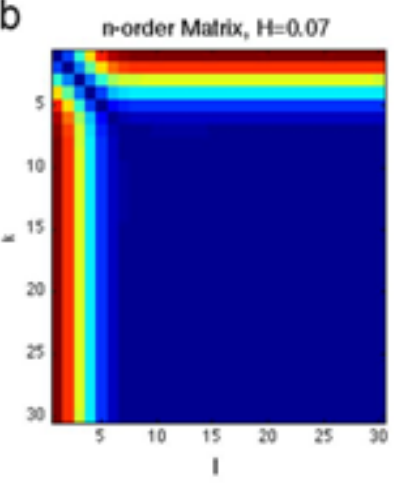

f

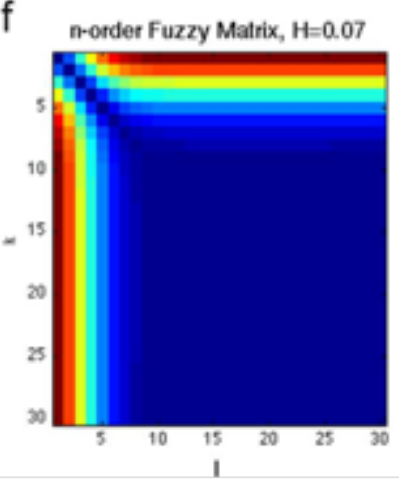

C

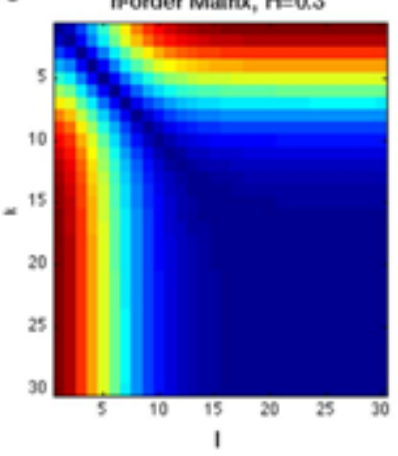

g

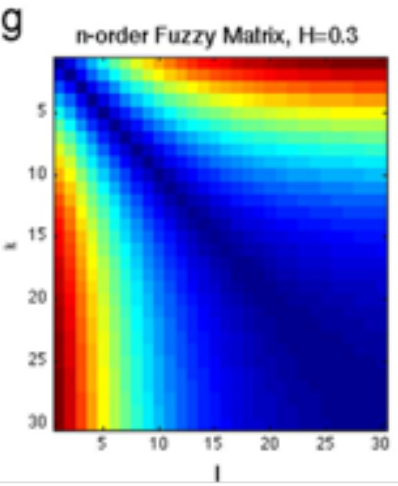

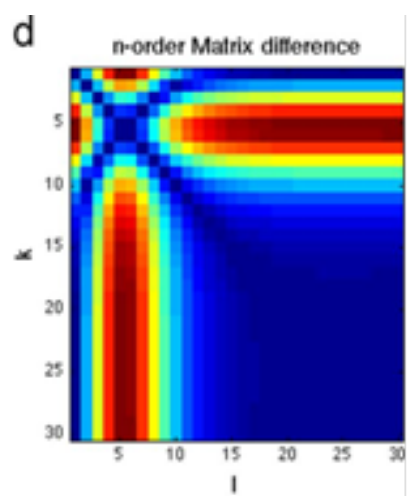

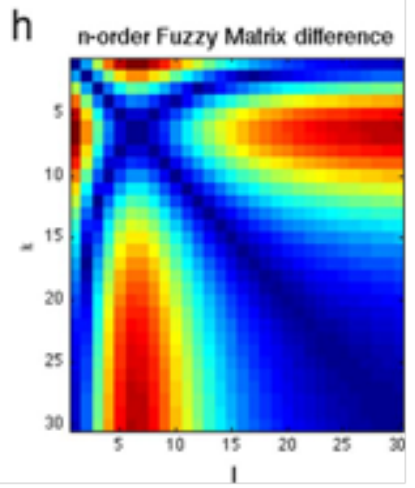

Figure 5: Representation of the matrix $\mathcal{M}_{p}^{(i)}$ for two different fBms. (a) Non-fuzzy estimations with $\Phi_{\infty}^{(1)}$ (blue line) and $\Phi_{\infty}^{(2)}$ (red line) with $\mathcal{H}=0.07$ and $\mathcal{H}=0.3$, respectively. (b) Non-fuzzy estimations with $\mathcal{M}_{\infty}^{(1)}$. (c) Non-fuzzy estimations with $\mathcal{M}_{\infty}^{(2)}$. (d) Non-fuzzy estimations with difference between $\mathcal{M}_{\infty}^{(1)}$ and $\mathcal{M}_{\infty}^{(2)}$. (e) Fuzzy estimations with $\Phi_{2}^{(1)}$ (blue line) and $\Phi_{2}^{(2)}$ (red line) with $\mathcal{H}=0.07$ and $\mathcal{H}=0.3$, respectively. (f) Fuzzy estimations with $\mathcal{M}_{2}^{(1)}$. (g) Fuzzy estimations with $\mathcal{M}_{2}^{(2)}$. (h) Fuzzy estimations with difference between $\mathcal{M}_{2}^{(1)}$ and $\mathcal{M}_{2}^{(2)}$. )

of $2.25 \mathrm{MHz}$ for an acoustic power limited to $1 \mathrm{~mW} / \mathrm{cm}^{2}$ (for more details see [28, 29]), was developed to measure both the FHR and fetal movements (pseudo-breathing, limb movements).

After locating the fetal heart with an echographic scanner, 80 Doppler recordings of 30 minutes each were acquired at CHRU Bretonneau Tours, France. In order to constitute homogeneous groups without spurious data, gestations complicated by other kinds of disorders (hypertension, diabetes) were discarded. Two groups of fetuses were selected: normal and those with severe Intra-Uterine Growth Retardation (IUGR). The normal group included 40 fetuses without disorders, delivered at term by spontaneous labor. The severe IUGR group included 40 fetuses delivered prematurely by cesarean section. For this clinical protocol, the gestational ages of fetuses ranged from 26th to 34th weeks. The consent of each parent was obtained and the study was approved by the Ethics Committee of the Clinical Investigation Centre for Innovative Technology of Tours (CIC-IT 1415 CHRU of Tours). All parents were over eighteen years of age and pregnancies were single.

\subsection{Results}

From our data set composed of 80 clinical recordings of 30 minutes, discrimination functions $\Delta_{\mathcal{E} p}(n, m)=\left|\mathcal{E}_{p}^{(1)}(n, m)-\mathcal{E}_{p}^{(2)}(n, m)\right|$ based on n-order similarity entropies $\mathcal{E}_{p}^{(i)}(n, m) \quad(i=1$ for normal and $i=2$ for abnormal fetuses) were calculated through a short term analysis using centered $\mathbf{X}$ vectors composed of 720 points and overlapping by $97 \%$. From each recording composed of 7200 points (sampling frequency of $4 \mathrm{~Hz}), 324$ non-fuzzy estimations $\mathcal{E}_{\infty}(n, m)$ and 324 fuzzy estimations $\mathcal{E}_{2}(n, m)$ were evaluated.

To evaluate fairly the levels of performance of the proposed methods, the dataset was randomly divided into a training set (20 from the normal group and 20 from the abnormal group) and a testing set (20 from the normal group and 20 from the abnormal group). 
From the training set, a total number of $2 \times 2 \times(40+40) \times 324$ of n-order entropies, (i) $\Delta_{\mathcal{M} \infty}$ were reported from non-fuzzy estimations (Table 4), ii) $\Delta_{\mathcal{M} 2}$ were evaluated from fuzzy estimations (Table 5), and iii) average values and relative accuracy of discrimination functions $\Delta_{\mathcal{E} p}(n, m)$ and $\Delta_{\mathcal{E} p}(1, m)$ (Table 6) were determined. From the training set, the optimal values of $m^{*}$ and $n^{*}$ were obtained (see Tables 3 and 4 ).

From the testing set, (i) boxplots (Fig. 6) and (ii) the sensitivity and specificity (see Table 7) were computed by using $m^{*}$ and $n^{*}$.

\begin{tabular}{cccccccc}
\hline $\mathrm{k}$ & $\mathrm{l}$ & & & & & & \\
\cline { 2 - 7 } & 1 & 2 & 3 & 4 & 5 & 6 & 7 \\
\hline 1 & 0.000 & 0.064 & 0.128 & 0.173 & $\mathbf{0 . 1 8 7}$ & 0.177 & 0.157 \\
2 & $\mathbf{0 . 0 6 4}$ & 0.000 & 0.064 & 0.109 & 0.123 & 0.113 & 0.092 \\
3 & 0.128 & 0.064 & 0.000 & 0.045 & 0.059 & 0.049 & 0.028 \\
4 & 0.173 & 0.109 & 0.045 & 0.000 & 0.014 & 0.004 & 0.016 \\
5 & $\mathbf{0 . 1 8 7}$ & 0.123 & 0.059 & 0.014 & 0.000 & 0.010 & 0.030 \\
6 & 0.177 & 0.113 & 0.049 & 0.004 & 0.010 & 0.000 & 0.020 \\
7 & 0.157 & 0.092 & 0.028 & 0.016 & 0.030 & 0.020 & 0.000 \\
\hline
\end{tabular}

Table 4: Discrimination functions $\Delta_{\mathcal{M} \infty}$ from non-Fuzzy estimations derived from FHR signals with $k, l$ ranging from 1 to 7 . Values in italic values correspond to 1-order similarity entropies. Values in bold correspond to the maximum of n-order similarity entropies.

\begin{tabular}{cccccccc}
\hline $\mathrm{k}$ & $\mathrm{l}$ & \multicolumn{1}{c}{3} & & & & \\
\cline { 2 - 7 } & 1 & 2 & 3 & 4 & 5 & 6 & 7 \\
\hline 1 & 0.000 & $\mathbf{0 . 0 6 3}$ & 0.122 & 0.164 & 0.186 & $\mathbf{0 . 1 9 1}$ & 0.186 \\
2 & $\mathbf{0 . 0 6 3}$ & 0.000 & 0.059 & 0.101 & 0.123 & 0.128 & 0.123 \\
3 & 0.122 & 0.059 & 0.000 & 0.042 & 0.064 & 0.069 & 0.064 \\
4 & 0.164 & 0.101 & 0.042 & 0.000 & 0.022 & 0.027 & 0.022 \\
5 & 0.186 & 0.123 & 0.064 & 0.022 & 0.000 & 0.005 & 0.000 \\
6 & $\mathbf{0 . 1 8 7}$ & 0.128 & 0.069 & 0.027 & 0.005 & 0.000 & 0.005 \\
7 & 0.186 & 0.123 & 0.064 & 0.022 & 0.000 & 0.005 & 0.000 \\
\hline
\end{tabular}

Table 5: Discrimination functions $\Delta_{\mathcal{M} 2}$ from fuzzy estimations derived from FHR signals with $k, l$ ranging from 1 to 7. Values in italic correspond to 1-order similarity entropies. Values in bold correspond to the maximum of n-order similarity entropies.

It can be seen in Tables 3 and 4 that the lowest values belonged to the 1-order entropy estimations. The best values of $\Delta_{\mathcal{E} p}\left(n^{*}, 1\right)$ and $\Delta_{\mathcal{E} p}\left(1, m^{*}\right)$ are reported in bold in Tables 4 and 5 . The maximum values of 1 -order entropies corresponded to $\Delta_{\mathcal{E} \infty}^{*}(1,2)$ and $\Delta_{\mathcal{E} 2}^{*}(1,1)$. It was obvious that the best values did not correspond to the 1-order entropies since the maxima corresponded to $\Delta_{\mathcal{E} \infty}(4,1)$ and $\Delta_{\mathcal{E} 2}(5,1)$ for non-fuzzy and fuzzy estimations, respectively. This outcome confirmed that it was preferable to use n-order rather than 1-order based-estimators of complexity.

The results shown in Table 6 demonstrated that it was preferable to use fuzzy algorithms since (i) the relative precision was lower for fuzzy than for non-fuzzy estimations and ii) average values were similar for fuzzy and non-fuzzy estimations, and no bias was introduced.

The findings shown in Fig. 6 demonstrated that meaningful classifications (Kruskal-Wallis test was performed with a p-value $<5 \%$ ) were obtained for all methods used. Note a small advantage with fuzzy estimations since outliers (green crosses in Fig. 6) were removed, showing that here the fuzzy approach improved statistical instability. It can be seen from Fig. 6 that the level of similarity was lower for abnormal fetuses than for normal fetuses. This outcome corroborated the results 


\begin{tabular}{ccccc}
\hline & & $\Delta_{\mathcal{E} p}(1,1)$ & $\Delta_{\mathcal{E} p}(4,1)$ & $\Delta_{\mathcal{E} p}(5,1)$ \\
\hline Non-fuzzy & Average & 0.06 & 0.19 & 0.18 \\
& Relative accuracy $(\%)$ & 46 & 52 & 55 \\
Fuzzy & Average & 0.06 & 0.19 & 0.19 \\
& Relative accuracy (\%) & 45 & 49 & 52 \\
\hline
\end{tabular}

Table 6: Average values and "relative accuracy" of discrimination functions $\Delta_{\mathcal{E} p}(n, m)$ derived from FHR signals. Values in parentheses are a kind of "relative accuracy" that represents differences in values of confidence interval $(75 \%)$ normalized by the average value. Fuzzy estimations were valuable for reducing estimation fluctuations.

previously reported in [3]. Furthermore, it can be seen that the similarity measurements were fairly homogeneous. This was certainly due to the good homogeneity of the fetus groups. Note that the high levels of performance of n-order entropy were confirmed by the very good values for sensitivity and specificity reported in Table 7 .

The findings reported in Table 7 showed that the sensitivity and specificity were good since they were above $86 \%$. This outcome was confirmed by the small values of the 5 -fold cross validation errors obtained with the linear discriminant analysis [31]. Fuzzy estimations were preferable since slightly improved sensitivity, specificity and cross validation error were found. The best performance was obtained for $\Delta_{\mathcal{E} \infty}^{*}(5,1)$, suggesting that n-order fuzzy entropies were better than 1-order fuzzy entropies.

To sum up this section, it was again shown that it was advantageous to use maximum n-order entropy rather than 1-order entropy. A slight advantage was found for the fuzzy approach. Moreover, all methods tested showed significant discrimination, which is valuable for improving medical diagnosis.

\begin{tabular}{cccccc}
\hline & & $\Delta_{\mathcal{E} p}(1,1)$ & $\Delta_{\mathcal{E} p}^{*}(1,2)$ & $\Delta_{\mathcal{E} p}^{*}(4,1)$ & $\Delta_{\mathcal{E} p}^{*}(5,1)$ \\
\hline \multirow{2}{*}{ Non-fuzzy } & Sensitivity (\%) & $86.9 \%$ & $87.2 \%$ & $88.3 \%$ & 89.4 \\
& Specificity (\%) & $86.9 \%$ & $87.2 \%$ & $88.3 \%$ & 89.4 \\
\multirow{5}{*}{ Fuzzy } & Cross validation error & 0.13 & 0.12 & 0.09 & 0.08 \\
& Sensitivity (\%) & $87.5 \%$ & $88.6 \%$ & $89.5 \%$ & 90.5 \\
& Specificity (\%) & $87.5 \%$ & $88.6 \%$ & $89.5 \%$ & 90.5 \\
& Cross validation error & 0.11 & 0.10 & 0.08 & 0.07 \\
\hline
\end{tabular}

Table 7: Sensitivity of discrimination functions $\Delta_{\mathcal{E} p}(n, m)$ calculated from FHR signals. Note that $\Delta_{\mathcal{E} p}^{*}(n, 1)$ was equal to $\Delta_{\mathcal{E} \infty}^{*}(4,1)$ for non-fuzzy estimations and to $\Delta_{\mathcal{E} 2}^{*}(5,1)$ for fuzzy estimations. Fuzzy estimations were of benefit since a slight improvement in both sensitivity and specificity can be seen.

\section{Discussion and conclusion}

The aim of this paper was to propose new descriptors to improve the differentiation between time series of differing complexity. The primary objective was to extend the complexity studies of time series recently undertaken in this field based on a search for the best parameter settings. This search for solutions revealed several original outcomes. The first original result was obtained by investigating the role played by the size pattern $m$. This led to the concept of maximum entropy $\mathcal{E}^{*}$ that we found to be related to the intrinsic feature $\mathcal{H}$ of the time series examined, i.e. fBm. As the concept of maximum entropy also explained the presence of slope changes in the function $\Phi(\mathrm{m})$ and as the 1-order entropy was the discrete derivative of the function $\Phi(m)$, then the presence of inflection points in the 1-order entropy led naturally to the concept of the $n$-order derivative that we have presented here as n-order entropy. This constitutes the second original finding. By adapting 

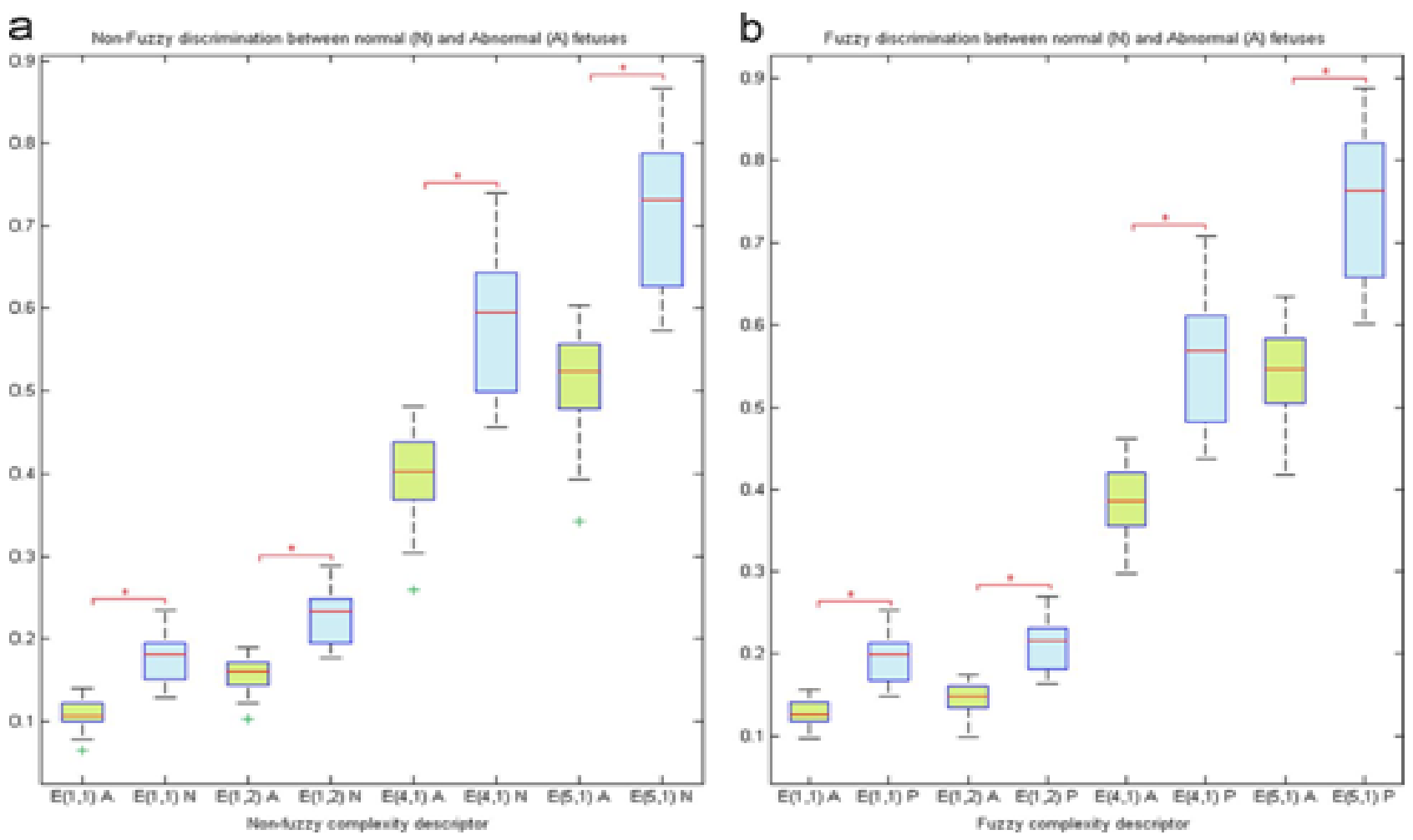

Figure 6: Boxplots of 1-order entropies and n-order entropies calculated from FHR signals. Left panel non-fuzzy estimations. Boxplots of 1 -order entropies $\mathcal{E}(1,1), \mathcal{E}^{*}(1,2)$, and n-order entropies $\mathcal{E}(4,1), \mathcal{E}(5,1)$ for normal (in green) and abnormal fetuses (in blue). Right panel fuzzy estimations. Boxplots of 1-order entropies $\mathcal{E}(1,1), \mathcal{E}^{*}(1,2)$ and n-order entropies $\mathcal{E}(4,1), \mathcal{E}(5,1)$ for normal (in green) and abnormal fetuses (in blue). Green crosses represent the presence of outliers. Red stars indicate that the two groups were significantly different (Kruskal-Wallis tests with p-value $<5 \%)$.

the concept of maximum entropy to n-order entropy and by combining the latter concept to the fuzzy concept, a very high performance level descriptor was then proposed.

Thus, four main important results were obtained in this study.

The first main result was derived from the research for the best setting in relation to pattern size $m^{*}$ when the tolerance $\mathrm{r}$ was constant and fixed at $r=0.2$. As a follow-up of previous studies by $[24,19]$, and based on the research for the best setting $r^{*}$ to maximize entropy $\mathcal{E}_{*}=\mathcal{E}\left(r^{*}\right)$, our findings showed that it was also of benefit to find the pattern size $m^{*}$ that maximized entropy $\mathcal{E}^{*}=\mathcal{E}\left(1, m^{*}\right)$. In particular, it was shown that both $m^{*}$ and $\mathcal{E}\left(1, m^{*}\right)$ were related to intrinsic features such as the Hurst exponent of fractional Brownian motion and we were very surprised to find very high values for pattern size $m^{*}$ since values lower than 20 are often reported [19]. In any event, it was shown that it was quite easy to discriminate fBms differing by at least $10 \%$ from the Hurst exponent considered. This outcome augurs well for detecting complexity changes of other nonlinear dynamic systems or for discriminating other kinds of signals of different complexity. Finding the best setting of $\mathrm{m}$ was interesting since it provided a complementary insight into the role of $m$ that was now no longer limited to the role of embedding dimension. It can therefore be confirmed that it is necessary to seek both the value of $m^{*}$ that maximizes the entropy and the value of the maximum entropy $\mathcal{E}\left(1, m^{*}\right)$. Nevertheless, the combined search for $r^{*}, m^{*}$ that would maximize the entropy $\mathcal{E}_{*}^{*}=\mathcal{E}\left(r^{*}, m^{*}\right)$ is a challenge that needs to be overcome and this will be addressed in a future paper.

The second main result was linked to the development of a new paradigm that encompasses the 1-order similarity entropy. The development of this new concept used with benefit the monotonic decrease in the function $\Phi(m)$, a feature that has never been used to date. As for $m^{*}$ and $\mathcal{E}^{*}$, it was shown that the n-order similarity entropy quite easily discriminated fBms with low Hurst exponents. This outcome augurs well for discrimination of biomedical signals since it was reported in [12] that such low Hurst exponents are measured for fetal heart rate signals. 
The third important outcome concerned the validation of the different descriptors of complexity in the discrimination between normal and abnormal fetuses. Using the best discrimination function based on the n-order similarity entropy, it was shown that it was fairly easy to discriminate between fetuses without disorders and fetuses with IUGR. It seemed clear that it was really the difference in complexity between the two groups of fetuses that was the key point for good discrimination. However, although the n-order similarity entropy was very appealing, it was still difficult to identify exactly what led the n-order similarity entropy to outperform the other methods. It was probably a mixture of several elements based on (i) the monotonic decrease in the probability of finding similar patterns of size $m$, (ii) the maximum gap between similarity measurements of patterns of nonconsecutive size and (iii) other features that remain to be revealed. The high level of performance in terms of sensitivity and specificity obtained for all complexity descriptors used in this study was certainly due to the level of homogeneity of each group. Note also that the levels of performance of the new proposed descriptors were very close. This finding is not so surprising since it was

shown that FHR time series could be coarsely considered as fBm of Hurst exponents differing in $\delta \mathcal{H}=0.23(\mathcal{H}=0.07$ and $\mathcal{H}+\delta \mathcal{H}=0.30)$ and since it was shown in this study that the proposed descriptors could significantly differentiate $\mathrm{fBm}$ of Hurst exponents differing at least by $\delta \mathcal{H}=0.01$. Nevertheless, although the results were very good, it is important that this study be extended to greater numbers of recordings. In any event, these findings augur well for the discrimination of other kinds of biomedical signals.

The final important finding was that it was almost always advantageous to develop descriptors with fuzzy foundations. Indeed, the use of a membership function led to reduction of the standard deviation of estimations, improvement in the relative precision and improvement in both the sensitivity and the specificity of the discriminator of fetal behaviors.

Above all, in antenatal medicine, our contributions complement contribution to the characterization of pathological IUGR and we hope that this contribution will reduce the risk of hypoxia often associated with increased morbidity and mortality in pre-mature neonates [30].

\section{Acknowledgements}

This study was supported financially by the "Agence Nationale de la Recherche" (Project ANR07-TECSAN-023, Surfoetus), by the National Council for Scientific Research in Lebanon (CNRS-L), Lebanese University and Imaging and Brain Laboratory, National Institute of Health and Medical Research, INSERM U930 in Tours, France. Furthermore, the authors would like to thank the Clinical Investigation Centre for Innovative Technology of Tours (CIC-IT 1415 CHRU of Tours) and Prof. F. Perrotin's team in the Obstetrics Department for their support in recording the signals.

\section{References}

[1] Marwan N., Wessel N., Meyerfeldt U., Schirdewan A. , Kurths J., Recurrence-plot-based measures of complexity and their application to heart-rate-variability data, Physical Review E, (66), (2002), 026702.

[2] Brodsky D., Chritsou H., Current concepts in intrauterine growth restriction, J. Intensive Care Med.,19 (6), (2004),307-319.

[3] Ferrario, M., Signorini, M., Magenes, G., Complexity analysis of the foetal heart rate variability: early identification of severe intrauterine growth-restricted foetuses, Med. Biol. Eng. Comput., 47, (2009), 911-919.

[4] Gough, N.A., Fractal analysis of foetal heart rate variability, Physiol. Meas.,14, (1993), 309-315.

[5] Felgueiras, C.S., de Sa, J.P., Bernardes, J. , Gamma, S., Classification of foetal heart rate sequences based on fractal features, Med. Biol. Eng. Comput.,36, (1998), 197-201. 
[6] Girault, J.-M., Kouame, D, Ouahabi, A, Analytical formulation of the fractal dimension of filtered stochastic signals, Signal Processing, 90, (2010), 2690-2697.

[7] Kikuchi, A., Unno, N., Horikoshi, T., Shimizu, T., Kozuma, S., Taketani, Y., Changes in fractal features of foetal heart rate during pregnancy, Early Hum. Dev.,81, (2005), 655-661.

[8] Ivanov.P.C, Amaral.L.A.N, Goldberger.A.L, Havlin.S, Rosenblum.M.G, Struzik.Z.R and Stanley.H.E, Multifractality in human heartbeat dynamics, Nature (London),5, (1999), 399-461.

[9] Wang, J., Ning, X., Ma, Q., Bian, C., Xu, Y., Chen, Y, Multiscale multifractality analysis of a 12-lead electrocardiogram, Phys. Rev. E, 71, (2005), 062902.

[10] Sassi R., Signorini M., Cerutti S., Multifractality and heart rate variabilty, Chaos, 19, (2009), 028507.

[11] Humeau A., Buard B., Mahe G., Chapeau-Blondeau F., Rousseau D., Abraham P., Multifractal analaysis of heart rate variability and laser Doppler flowmetry fluctuations: comparison of results from different numerical methods, Physics in Medicine and Biology, 55, (2010), 62796297.

[12] Oudjemia S., Zaylaa A., Haddab S., Girault J.-M. Coarse-grained multifractality analysis based on structure function measurements to discriminate healty from distressed foetuses, Computational and Mathematical Methods in Medecine, (2013), ID 152828, 9 pages.

[13] Mohebbi M. , Ghassemian H. , and Asl B. M. , Structures of the Recurrence Plot of Heart Rate Variability Signal as a Tool for Predicting the Onset of Paroxysmal Atrial Fibrillation, J. Med Signals Sens., 1(2), (2011), 113-121.

[14] Pincus, S.M., Viscallero, R.R., Approximate entropy: a regularity measure for foetal heart rate analysis, Obstet. Gynecol., 79,(1992),249-255.

[15] Ferrario M., Signorini M., Magenes M., Cerutti S., Comparison of Entropy-Based Regularity Estimators: Application to the foetal Heart Rate Signal for the Identification of foetal Distress, IEEE T Bio-Med Eng, 53, (2006), 119-125.

[16] Chen W.T., Zhuang J., Yu W.X., Wang Z.Z., Measuring complexity using FuzzyEn, Apen and SampEn, Med. Eng. Phys., 31, (2009), 61-68.

[17] Liu C.Y., Liu C.C., Shao P., Li L.P., Sun X., Wang X.P., Liu F., Comparison of different threshold values $\mathrm{r}$ for approximate entropy: application to investigate the heart rate variability between heart failure and healthy control group, Physiol. Meas., 32 (2), (2011), 168-180.

[18] Liu C., Li K., Liu L., Zheng D., Liu C., Liu S., Analysis of heart rate variability using fuzzy measure entropy, Computers in Biology and Medicine, 43, (2013), 100-108.

[19] Restrepo J.F., Schlotthauer G., Torres M.E., Maximum approximate entropy and r threshold: a new approach for regularity changes detection, Physica A, 409, (2014), 97-109.

[20] Boskovic A., Loncar-Turukalo T., Sarenac O., Japundzic-Zigon N., Bajic D. Unbiased entropy estimates in stress: A parameter sutdy, Com. Biol. Med,42, (2012), 667-679.

[21] Chon K.H., Scully C., Lu S., Approximate entropy for all signals, IEEE Eng. Med.Biol. Mag., 28, (2009), 18-23.

[22] Pincus S., Gladstone L., Ehrenkranz R., A regularity statistic for medical data analysis, J. Clin. Med. Monit., 4, (1991), 335-345.

[23] Pincus S.M., Keefe D.L. , Quantification of Hormone pulsatility via an approximate entropy algorithm, Amer. J. Physiol. Endocrinol. Metabol., 262, (1992), E741-E754. 
[24] Lu S., Chen X., Kanters J.K., Solomon I.C., Chon K.H., Automatic selection of the threshold value $\mathrm{r}$ for approximate entropy, IEEE Trans. Biomed. Eng., 55, (2008), 1966-1972.

[25] Kennel M.B, Brown R., Abarbanel H.D.I., Determining Embedding dimension for phase-space reconstruction using geometrical construction, Phys. Rev. A, 6, (1992), 3403-3411.

[26] Cao L., Practical method for determining the minimum embedding dimension of a scalar time series, Physica D,110, (1997), 43-50.

[27] Pincus S.M., Goldberger A.L. , Physiological time series analysis: what does regularity quantify, Amer. J. Physiol. Heart Circul. Physiol., 266,(1994), E1643-E1656.

[28] Voicu, I., Girault, J.-M., Menigot, S., Improved estimation of the foetal heart rate using directional Doppler signal and YIN, IRBM, 33, (2012), 262-270.

[29] Voicu, I., Menigot, S., Kouamé D., Girault, J.-M., New estimators and guidelines for better use of foetal heart rate estimators with Doppler ultrasound devices, Comput. Math. Methods Med., (2014), ID 784862, 10 pages.

[30] T.J. Garite, R. Clark, J.A. Thorp, Intrauterine growth restriction increases morbidity and mortality among premature neonates, Am. J. Obstet. Gynecol., 191(2), (2004), 481-487.

[31] T. Hastie, R. Tibshirani, J. Friedman, The Elements of Statistical Learning, Springer, New York, 2001. 